OPEN ACCESS

Edited by:

Changhua Wang,

Wuhan University, China

Reviewed by:

Ying Zhang,

First Bethune Hospital of Jilin University, China

Paula Alexandra Oliveira,

Universidade de Trás-os-Montes e

Alto Douro, Portugal

*Correspondence:

Xiao-Wei Li

793601136@qq.com

†These authors have contributed equally to this work

Specialty section:

This article was submitted to Cardiovascular and Smooth Muscle

Pharmacology,

a section of the journal

Frontiers in Pharmacology

Received: 08 March 2018 Accepted: 07 September 2018 Published: 27 September 2018

Citation:

Zheng $C$, Lin J-F, Lin Z-H, Lin W-Q,

Thapa S, Lin Y-Z, Lian H, Liu Z-R, Chen J-H and Li X-W (2018) Sodium Houttuyfonate Alleviates Post-infarct Remodeling in Rats via AMP-Activated Protein Kinase

Pathway. Front. Pharmacol. 9:1092. doi: 10.3389/fphar.2018.01092

\section{Sodium Houttuyfonate Alleviates Post-infarct Remodeling in Rats via AMP-Activated Protein Kinase Pathway}

\author{
Cheng Zheng ${ }^{1 t}$, Jia-Feng Lin ${ }^{1 t}$, Zhong-Hao Lin', Wei-Qian Lin', Saroj Thapa', \\ Yuan-Zheng Lin', Hao Lian', Zhi-Rui Liu', Jia-Hui Chen² and Xiao-Wei Li' ${ }^{*}$ \\ 1 Department of Cardiology, The Second Affiliated Hospital and Yuying Children's Hospital of Wenzhou Medical University, \\ Wenzhou, China, ${ }^{2}$ Department of Cardiology, Taishun General Hospital, Wenzhou, China
}

With the chronic ischemia persisting after acute myocardial infarction, the accompanying low-degree inflammation and subsequent fibrosis result in progression of cardiac remodeling and heart failure. Recently, Sodium Houttuyfonate (SH), a pure compound extracted from Houttuynia cordata, has been confirmed exerting anti-inflammatory and anti-fibrotic effects under diseased situations. Here, we aimed to investigate whether $\mathrm{SH}$ could reverse the cardiac remodeling post-myocardial infarction by alleviating cardiac inflammation and fibrosis. Left anterior descending coronary artery of adult male Sprague-Dawley rats was ligated to elicit myocardial infarction. Low and high dose of SH was administered by oral gavage for four consecutive weeks post-myocardial infarction. Long-term SH treatment decreased heart rate, heart weight/ body weight (HW/BW), and left ventricle weight/body weight (LWW/BW), reduced cardiac expression of brain natriuretic peptide (BNP), improved left ventricular heart function, and ameliorated the histopathological changes caused by myocardial infarction. Western blotting revealed the expression of tumor necrosis factor- $\alpha$ (TNF- $\alpha$ ), interleukin-6 (IL-6), transforming growth factor- $\beta$ (TGF- $\beta$ ), collagen I, and collagen III of the infarcted ventricle were reduced by $\mathrm{SH}$ treatment. Meanwhile, we found that $\mathrm{SH}$ treatment post-myocardial infarction activated AMP-activated protein kinase (AMPK) and suppressed nuclear

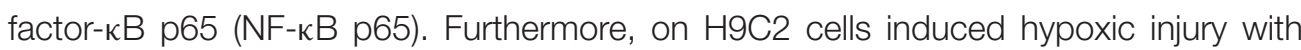
cobalt chloride $\left(\mathrm{CoCl}_{2}\right)$, the reduction of inflammatory cytokines (IL-6, TNF- $\alpha$, and TGF- $\beta$ ), activation of AMPK, and suppression of NF-kB p65 were also observed by $\mathrm{SH}$ treatment. However, transfection of $\mathrm{H} 9 \mathrm{C} 2$ with AMPK $\alpha$ siRNA blunted the suppression of NF-кB p65 and inflammatory cytokines (IL-6, TNF- $\alpha$, and TGF- $\beta$ ) by SH post-hypoxia. Taken together, these findings suggested that long-term administration of SH post-myocardial infarction reduced cardiac inflammatory and fibrotic responses, and reversed cardiac remodeling process. The underlying mechanism may be activating AMPK and suppressing NF-кB pathway.

Keywords: Sodium Houttuyfonate, myocardial infarction, post-infarct remodeling, AMP-activated protein kinase, cardiac inflammatory response, cardiac fibrotic response 


\section{INTRODUCTION}

The process of cardiac remodeling post-myocardial infarction initiated immediately after acute myocardial infarction and was defined as maladaptive responses in both infarcted and non-infarcted regions of the ventricle, leading to impaired contractile function, ventricular dilatation, and heart failure. Recent studies have revealed that the persisting low-degree inflammatory and fibrotic responses participated in the adverse cardiac remodeling process post-myocardial infarction and speeded up the development of chronic heart failure (Li et al., 2006; Takahashi et al., 2008; Hamid et al., 2011; Sobirin et al., 2012; Shivshankar et al., 2014; Wang B. et al., 2017). Thus, modulation of the inflammatory and fibrotic responses may be a promising therapy for adverse cardiac remodeling and heart failure post-myocardial infarction.

Sodium Houttuyfonate (SH), which was a pure compound extracted from a Chinese herb Houttuynia cordata, has shown potent anti-inflammatory and anti-fibrotic activities on various animal and cell models of diseases, like lipopolysaccharide (LPS)-induced acute lung injury (ALI), LPS-induced bovine endometrial epithelial cell inflammation, smoking-induced chronic obstructive pulmonary disease (COPD), ventricular remodeling induced by abdominal aortic banding and so on (Gao et al., 2010; Du et al., 2012; Kang and Koppula, 2014a, 2015; Xu et al., 2015; Zhu et al., 2015; Wu et al., 2017). Based on these findings, we doubted whether $\mathrm{SH}$ with an inherent anti-inflammatory and anti-fibrotic potency, could ameliorate post-infarct cardiac remodeling and heart failure.

Adenosine monophosphate-activated protein kinase (AMPK), which consisted of three subunits, $\alpha, \beta, \gamma$, that together made a functional enzyme, worked as an energy sensor to provide metabolic adaptations under the ATP-deprived conditions, such as ischemia and nutritional stress (Calvert et al., 2008; Quan et al., 2018). Activation of AMPK has been confirmed exerting pleiotropic functions against inflammatory response and tissue fibrosis and owning beneficial properties in a series of diseases (Peairs et al., 2009; Chiang et al., 2017; Kim et al., 2017). Intriguingly, recent studies have found activation of AMPK could be elicited or enhanced by $H$. cordata treatment in diseased situations (Peairs et al., 2009; Xu et al., 2015; Wang J.H. et al., 2017).

In this article, we mainly focused on investigating two unknown entities, whether $\mathrm{SH}$ treatment alleviated the cardiac remodeling post-myocardial infarction, and if so, whether AMPK activation was involved in the benefit of SH treatment.

\section{MATERIALS AND METHODS}

The experimental design of our study was presented in Figure 1.

\section{Reagents}

Sodium Houttuyfonate, Hematoxylin and Eosin (HE) stain kit and Mallory's trichrome stain kit were purchased from Solarbio Science \& Technology (Shanghai, China). The antibodies phospho-AMPK $\alpha$ (Thr172)(40H9)Rabbit mAb\#2535, $\mathrm{AMPK} \alpha(\mathrm{D} 63 \mathrm{G} 4)$ Rabbit mAb\#5832, NF-кB p65 (D14E12) $\mathrm{XP}^{\circledR}$ Rabbit mAb \#8242, Phospho-NF-кB p65 (Ser536) (93H1) Rabbit $\mathrm{mAb} \# 3033$ were purchased from Cell Signaling Technology (Danvers, MA, United States). The antibodies $\beta$-Actin (4D3) monoclonal mouse antibody\# BS6007M was

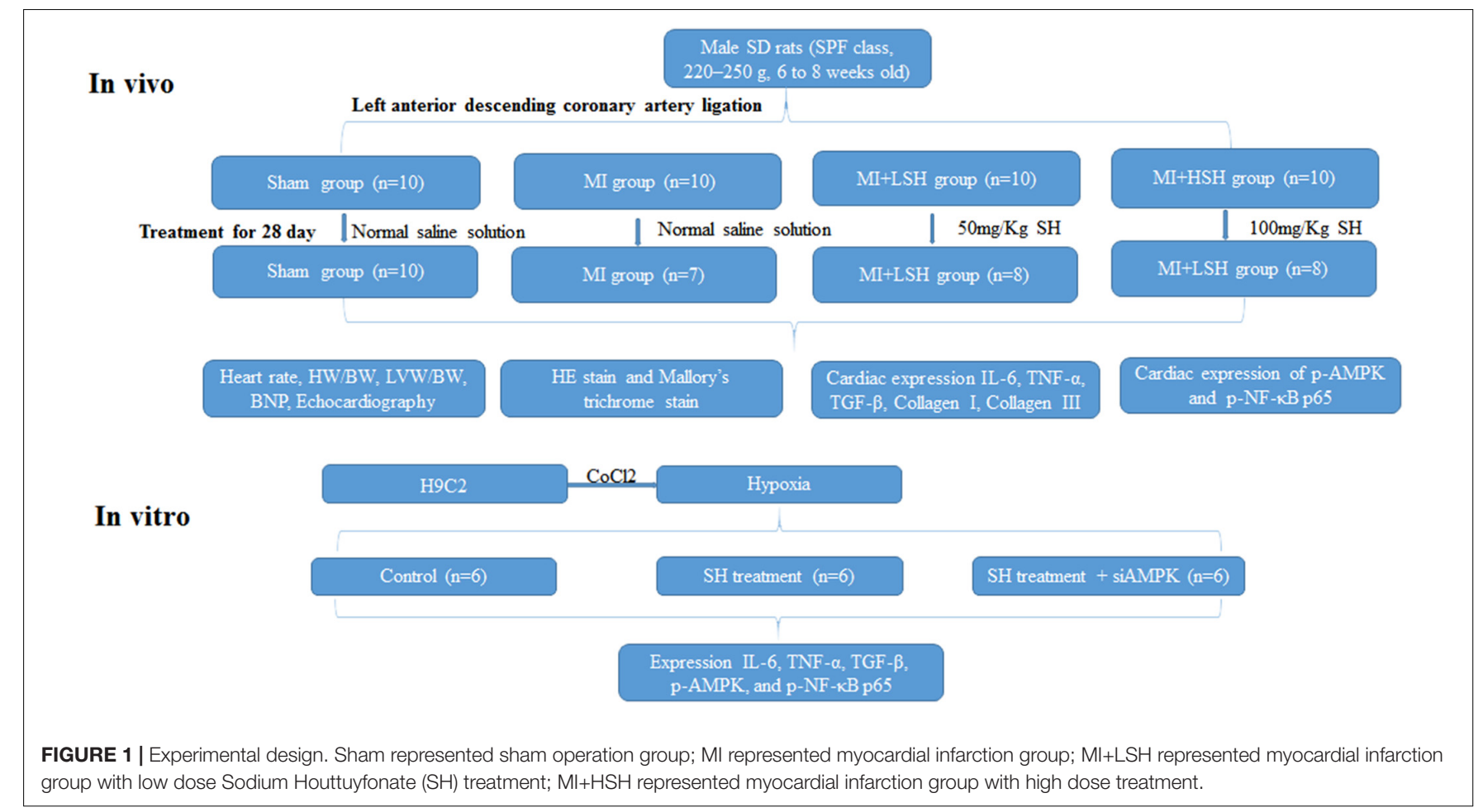


purchased from Bioworld Technology (Nanjing, China). The antibodies IL-6 mouse Antibody\#53324 was purchased from R\&D System Technology (Minneapolis, MN, United States). The antibodies tumor necrosis factor (TNF)- $\alpha \#$ Rabbit ab6671, Rabbit polyclonal to TGF $\beta 1 \#$ ab92486, Rabbit polyclonal to Collagen I\#ab34710, and Rabbit polyclonal to Collagen III\#ab7778 and Rabbit monoclonal [EPR16891] to GAPDH, anti-BNP antibody ab19645 were purchased from Abcam (Shanghai, China). Horseradish peroxidase (HRP) conjugated goat anti-rabbit IgG antibodies and anti-mouse IgG antibodies were purchased from Biosharp (Hefei, China). Cell counting kit-8 was purchased from Dojindo Molecular Technologies (Rockville, MD, United States). SiAMPK $\alpha$ was bought from Riobio technologies (Guangzhou, China). Lipofectamine ${ }^{\circledR}$ 2000 reagent was bought from Invitrogen (Carlsbad, CA, United States).

\section{Animal Preparation}

A total of 80 Male Sprague-Dawley rats (specific pathogen-free (SPF) class, 220-250 g, 6 to 8 weeks old) were purchased from Shanghai Laboratory Animal Center of China (Shanghai SLAC Laboratory Animal Co., Ltd.) and were kept under the SPF conditions $\left(24 \pm 1{ }^{\circ} \mathrm{C}, 45 \pm 10 \%\right.$ humidity $)$ with a 12-h light/dark cycle daily and food and water available ad libitum in the Wenzhou Medical University animal facilities. All animal experiments were approved by the Animal Ethics Committee of Wenzhou Medical University (number wydw2014-0058) and conformed to the Guide for the Care and Use of Laboratory Animals by the National Institutes of Health.

\section{Myocardial Infarction and Animal Model Establishment}

After induction of anesthesia (urethane $1.5 \mathrm{~g} / \mathrm{kg}$, administered intraperitoneally), the rats received left parasternotomy under transtracheal ventilation. After the hearts were deprived of pericardium and fully exposed, the left anterior descending coronary artery was visualized directly and ligated by a 6-0 Prolene suture at about $2 \mathrm{~mm}$ from its origin. All the operations adhered strictly to the aseptic techniques. Ten rats were randomly selected and received sham operation. All the other rats received the coronary artery ligation to induce myocardial infarction, the surviving rats after operation were then assigned blindly to three groups. The following treatments were given, respectively: sham-operated rats with normal saline solution administered by oral gavage once daily for 28 days (Sham, $n=10$ ), myocardial infarction rats with normal saline solution administered by oral gavage once daily for 28 days (MI, $n=10$ ), myocardial infarction rats with low dose $\mathrm{SH}$ of $50 \mathrm{mg} / \mathrm{Kg}$ administered by oral gavage once daily for 28 days ( $\mathrm{MI}+\mathrm{LSH}, n=10$ ), myocardial infarction rat with high dose $\mathrm{SH}$ of $100 \mathrm{mg} / \mathrm{Kg}$ administered by oral gavage once daily for 28 days $(\mathrm{MI}+\mathrm{HSH}, n=10)$. On the 28th day, all the surviving rats from each group were sacrificed and their hearts were obtained for histopathological and biochemical examinations. All rats were anesthetized (urethane $1.5 \mathrm{~g} / \mathrm{kg}$, administered intraperitoneally) prior to sacrifice. Until sufficient sedation was achieved, the chest of rats were opened and the hearts were obtained.

\section{Doppler Echocardiography Study}

Transthoracic echocardiography was performed on the 28th day after ligation with vevo1100 cardiovascular research ultrasound machine (Visualsonics, Japan). At the papillary muscle level, the LV end-diastolic diameter (LVEDd) and LV end-systolic diameter (LVESd) were measured by short-axis views of M-mode tracings from the anterior to posterior LV wall, the left ventricular ejection fraction (LVEF) was evaluated by the Simpson approach. All measurements were performed by an experienced technician who was blinded to the study groups.

\section{Heart Rate Calculation}

Prior to sacrifice, the heart rate of rats was recorded for $10 \mathrm{~min}$ by a computer-based electrical physiology system (PowerLab 8/36; AD Instruments, Colorado Springs, CO, United States).

\section{HW/BW and LVW/BW Calculation}

The heart weight (HW), left ventricle weight (LVW), body weight (BW), and the ratio of $\mathrm{HW}$ and $\mathrm{LVW}$ to $\mathrm{BW}$ (HW/BW and LVW/BW) were calculated on the 28th day.

\section{Hematoxylin and Eosin Stain and Mallory's Trichrome Stain}

The left ventricles of rats were obtained on the 28th day after ligation. LV middle ring (the middle $1 / 3$ of the left ventricle) was embedded in paraffin, sectioned into 5-um-thick slices and treated with HE stain and Mallory's trichrome stain, respectively. The infiltration of inflammatory cells and fibrotic scar tissue were observed at infarct zone, peri-infarct border zone (within $0.5-2 \mathrm{~mm}$ from infarct zone) and remote zone (within $2-3 \mathrm{~mm}$ from infarct zone).

\section{Western Blotting}

The proteins were extracted from the left ventricle and H9C2 cells. Each sample was separated by SDS-PAGE and transferred to a polyvinylidene difluoride membrane. The membrane was blocked in 5\% non-fat dry milk. After being incubated with the specific primary antibody (anti-phospho-AMPK $\alpha$, anti-AMPK $\alpha$, anti-NF-кB p65, anti-phospho-NF-кB p65, anti-IL-6, anti-TNF- $\alpha$, anti-TGF $\beta$, anti-collagen I, anti-collagen III, anti- $\beta$-actin, anti-GAPDH) and secondary antibody (HRP conjugated goat anti-rabbit and anti-mouse antibodies), the membrane was detected with the enhanced chemiluminescence detection system (Millipore, Billerica, MA, United States).

\section{Cellular Experiments}

The rat cardiomyoblast cell line, H9C2, was obtained from the Cell Bank of the Chinese Academy of Sciences (Shanghai, China) and cultured in Dulbecco's modified Eagle's medium containing $10 \%$ fetal bovine serum. The cells were maintained at $37^{\circ} \mathrm{C}$ in a humidified atmosphere with $5 \% \mathrm{CO}_{2}$. H9C2 cells (100 $\mathrm{\mu l} /$ well) were seeded into 96 -well plate at a density of 
TABLE 1 | Rat characteristics and cardiac function results from each group.

\begin{tabular}{|c|c|c|c|c|}
\hline Variable & Sham $(n=10)$ & MI $(n=7)$ & MI+LSH $(n=8)$ & MI+HSH $(n=8)$ \\
\hline \multicolumn{5}{|l|}{ Rat characteristics } \\
\hline Body weight, $g$ & $297.2 \pm 14.21$ & $291.28 \pm 18.48$ & $306.62 \pm 10.42$ & $301 \pm 16.14$ \\
\hline Heart weight, mg & $837.1 \pm 70.2$ & $1292.86 \pm 62.11^{*}$ & $1134.75 \pm 44.46^{* \#}$ & $1030.5 \pm 73.96^{* \# \wedge}$ \\
\hline Heart weight/body weight, mg/g & $2.83 \pm 0.33$ & $4.45 \pm 0.21^{*}$ & $3.71 \pm 0.22^{* \#}$ & $3.44 \pm 0.36^{* \# \wedge}$ \\
\hline Left ventricular weight, mg & $578.9 \pm 40.54$ & $917.14 \pm 40.48^{*}$ & $846.25 \pm 26.34^{* \#}$ & $730.5 \pm 39.53^{* \# \wedge}$ \\
\hline Left ventricular weight/body weight, mg/g & $1.95 \pm 0.18$ & $3.16 \pm 0.23^{*}$ & $2.76 \pm 0.14^{* \#}$ & $2.43 \pm 0.09^{* \# \wedge}$ \\
\hline \multicolumn{5}{|l|}{ Cardiac function } \\
\hline Heart rate, bpm & $390.8 \pm 10.65$ & $466.86+20.01^{*}$ & $430.12 \pm 9.43^{* \#}$ & $412 \pm 12.2^{* \# \wedge}$ \\
\hline LVEDd, mm & $5.15 \pm 0.55$ & $7.94 \pm 0.29 *$ & $7.50 \pm 0.26^{* \#}$ & $7.10 \pm 0.24^{* \# \wedge}$ \\
\hline LVESd, mm & $2.81 \pm 0.49$ & $6.58 \pm 0.19 *$ & $5.99 \pm 0.28^{* \#}$ & $5.40 \pm 0.39^{* \# \wedge}$ \\
\hline LVEF, \% & $83.52 \pm 5.03$ & $42.83 \pm 5.02^{*}$ & $48.90 \pm 4.37^{* \#}$ & $55.70+6.90^{* \# \wedge}$ \\
\hline
\end{tabular}

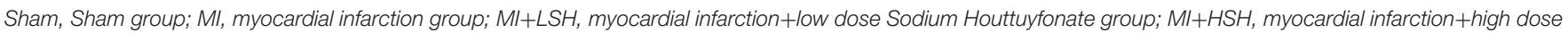
Sodium Houttuyfonate group; * compared with Sham group, $p<0.05$; ${ }^{\#}$ compared with MI group, $p<0.05$; ${ }^{\wedge}$ compared with MI+LSH group, $p<0.05$.

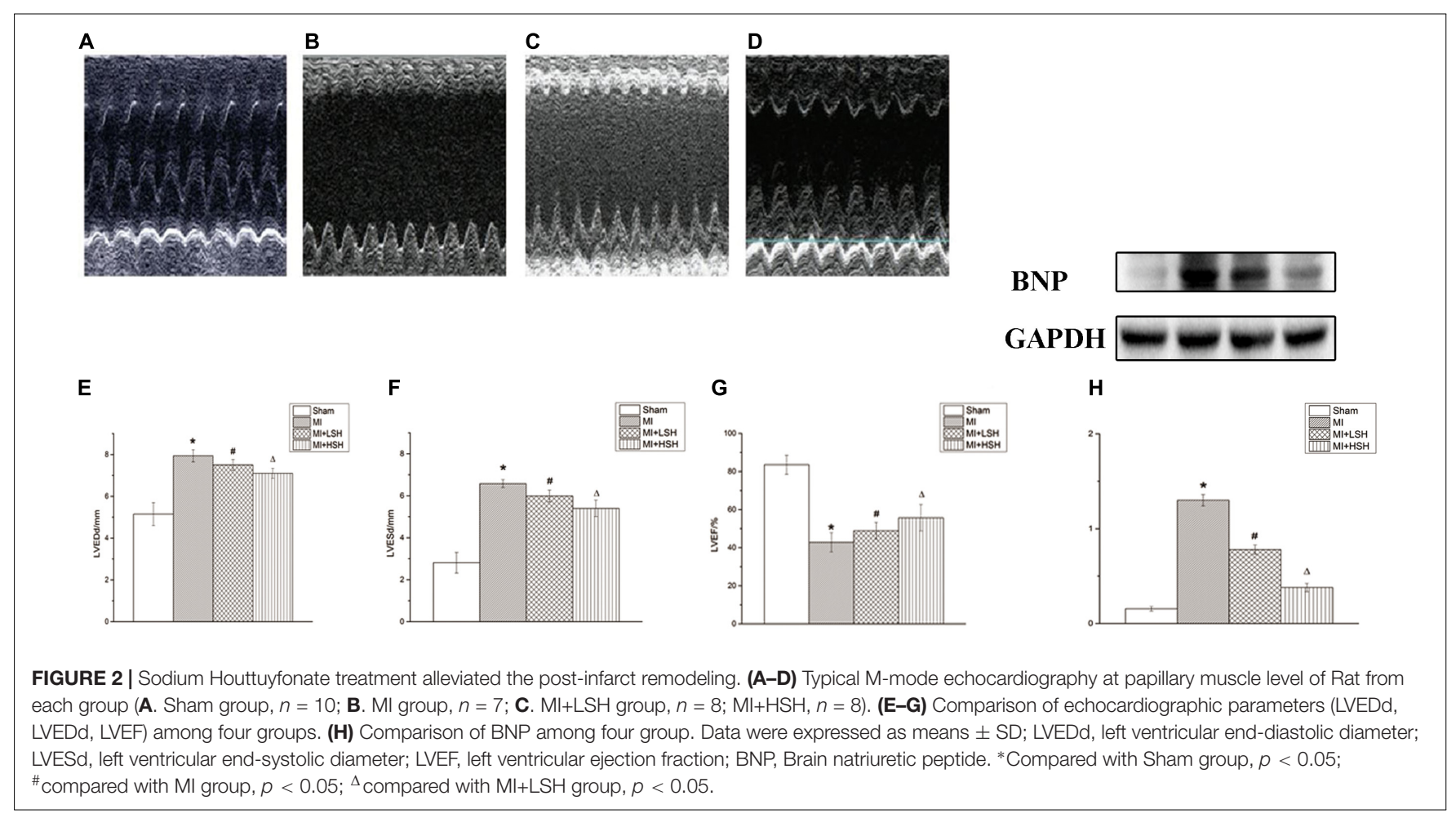

$3.0 \times 10^{4}$ cells $/ \mathrm{ml}$ and incubated for $24 \mathrm{~h}$ in medium containing different concentrations $(0,200,400,600,800,1000,1200$, and $14000 \mu \mathrm{M})$ of Cobalt chloride $\left(\mathrm{CoCl}_{2}\right)$. Cell viability was tested with cell counting kit- 8 , the concentration of $\mathrm{CoCl}_{2}$ resulting in half reduction of the cell viability was chosen to induce chemical hypoxia. In addition, H9C2 cells were seeded into 96 -well plate at a density of $3.0 \times 10^{4}$ cells $/ \mathrm{ml}$ and incubated for $24 \mathrm{~h}$ in medium in the presence of different concentrations $(0,10,20,30,40,50$, 60,70 , and $80 \mu \mathrm{g} / \mathrm{ml}$ ) of SH. Cell viability was also evaluated with $\mathrm{CCK}-8$, the concentrations of $\mathrm{SH}$ resulting in a reduction of the cell viability was excluded. In order to verify the most appropriate concentration of $\mathrm{SH}$ for $\mathrm{H} 9 \mathrm{C} 2$ cells post-hypoxia, the H9C2 were induced hypoxia first and then treated with different concentrations of $\mathrm{SH}$, which were in the range of no cell viability reduction. Changes in protein expression were measured by western blotting.

Moreover, to verify whether the effect of SH on H9C2 cells post chemical hypoxia was related to AMPK, siRNA-mediated knockdown of AMPK $\alpha$ was performed. H9C2 cells were transfected with siAMPK $\alpha$ by using Lipofectamine ${ }^{\circledR} 2000$ reagent for $6 \mathrm{~h}$ at $37^{\circ} \mathrm{C}$, then treated with $\mathrm{CoCl}_{2}$ for $24 \mathrm{~h}$ and incubated with fresh cell culture medium containing the most appropriate $\mathrm{SH}$ for the next $24 \mathrm{~h}$. Changes in protein expression were measured by western blotting.

\section{Statistical Methods}

Statistical analyses were performed using SPSS 14 software (Unicom, Mission Hills, CA, United States). Data were 
expressed as mean \pm standard deviation. Shapiro-Wilk test and Kolmogorov-Smirnov test were used for normal distribution. If distribution of the data is normal, outcomes are compared among groups using 1-way ANOVA followed by the Dunnett multiple-comparison test. If not, Wilcoxon's rank sum test should be applied instead. A value of $p<0.05$ was considered significant.

\section{RESULTS}

\section{Sodium Houttuyfonate Treatment Did Not Improve the Survival of Rats Post-myocardial Infarction, However, It Reduced Heart Rate, HW/BW and LVW/BW}

In this study, 10 rats received sham operation and were included in the Sham group. The other 70 rats were all referred to the procedure of left anterior descending coronary artery ligation, and only 37 rats survived the operation. Thirty rats were randomly selected from the 37 surviving rats and assigned blindly to three groups (MI, MI+LSH, MI+HSH groups) with 10 rats in each group.

We first investigated the effect of $\mathrm{SH}$ treatment in terms of survival analysis during the 28-day follow-up. In MI, MI+LSH and $\mathrm{MI}+\mathrm{HSH}$ group, 7 rat deaths occurred within the first 3 days after myocardial infarction, 5 rats were supposed died of heart failure with the presence of edematous lungs and 2 rats died from myocardial rupture. There was no obvious survival difference among MI, MI+LSH and MI+HSH groups (MI 70\% vs. MI+LSH $80 \%$ vs. MI+HSH $80 \%, p>0.05$ ), with three deaths in MI group, two deaths in both MI+LSH and MI+HSH groups. No animal death was observed in Sham group.

We also investigated whether $\mathrm{SH}$ treatment influenced heart rate $(\mathrm{HR}), \mathrm{HW} / \mathrm{BW}$ and $\mathrm{LVW} / \mathrm{BW}$ of rats on the 28th day post-myocardial infarction. Compared with Sham group, rats in the MI group presented an increased HR, HW/BW and LVW/BW $(p<0.05)$, while SH treatment decreased the HR, HW/BW, and LVW/BW of the infarcted rats $(p<0.05)$ and more reduction of these parameters was observed in MI+HSH group, see Table 1.

\section{Administration of Sodium Houttuyfonate Improved Left Ventricular Function, Decreased Left Ventricular Diameters and Cardiac Expression of Brain Natriuretic Peptide (BNP) of Rats Post-myocardial Infarction}

We further used three echocardiographic parameters, including LVEDd, LVESd, and LVEF, to evaluate the cardiac remodeling post-myocardial infarction. On the 28th day, we found that compared with rats from Sham group, there was an obvious adverse cardiac remodeling occurred on rats from MI group with enlarged left ventricle size and decreased left ventricular ejection function $(p<0.05)$. However, decreased left ventricular end-diastolic and end-systolic diameters with increased left ventricle ejection fraction was observed in $\mathrm{SH}$ treatment groups
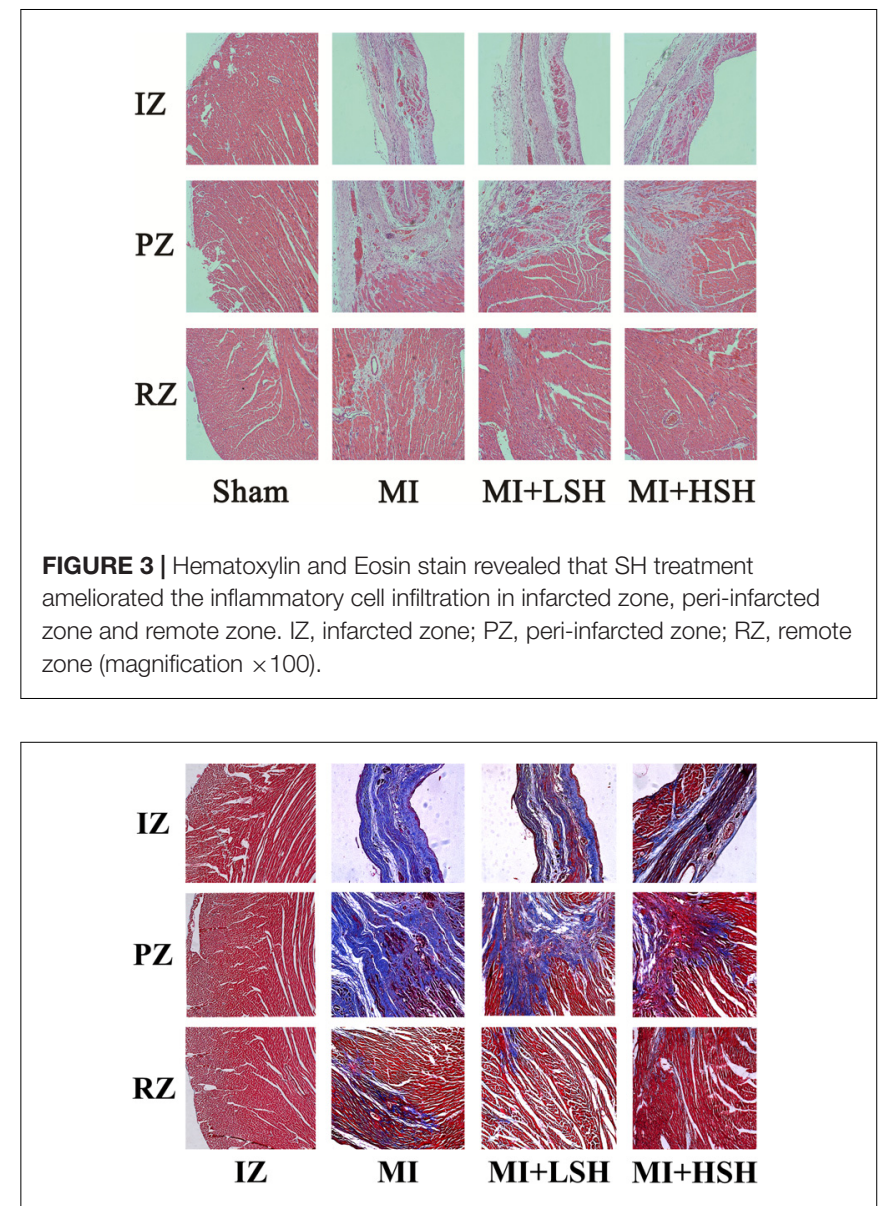

FIGURE 4 | Mallory's trichrome stain revealed that SH treatment ameliorated the fibrosis infiltration in infarcted zone, peri-infarcted zone, and remote zone. $I Z$, infarcted zone; PZ, peri-infarcted zone; RZ, remote zone (magnification $\times 100$ ).

$(p<0.05)$. In addition, the improvement of echocardiographic parameters were more obvious in the MI+HSH group $(p<0.05)$, as shown in Table 1 and Figure 2.

Brain natriuretic peptide (BNP) has been proved useful in the diagnosis of cardiac dysfunction and heart failure. Therefore, we also explored the effect of $\mathrm{SH}$ treatment on cardiac BNP level post-myocardial infarction. We found that compared with rats from Sham group, a remarkable elevation of cardiac BNP was observed in MI group $(p<0.05)$. SH treatment groups reduced the expression of BNP from the infarcted heart $(p<0.05)$. Additionally, the cardiac BNP were more reduced by high dose SH treatment $(p<0.05)$, see Figure 2.

\section{Administration of Sodium Houttuyfonate Alleviated the Infiltration of Inflammation and Fibrosis in the Left Ventricle Post-myocardial Infarction}

We next examined the effect of $\mathrm{SH}$ treatment on cardiac histopathological changes post-myocardial infarction. 

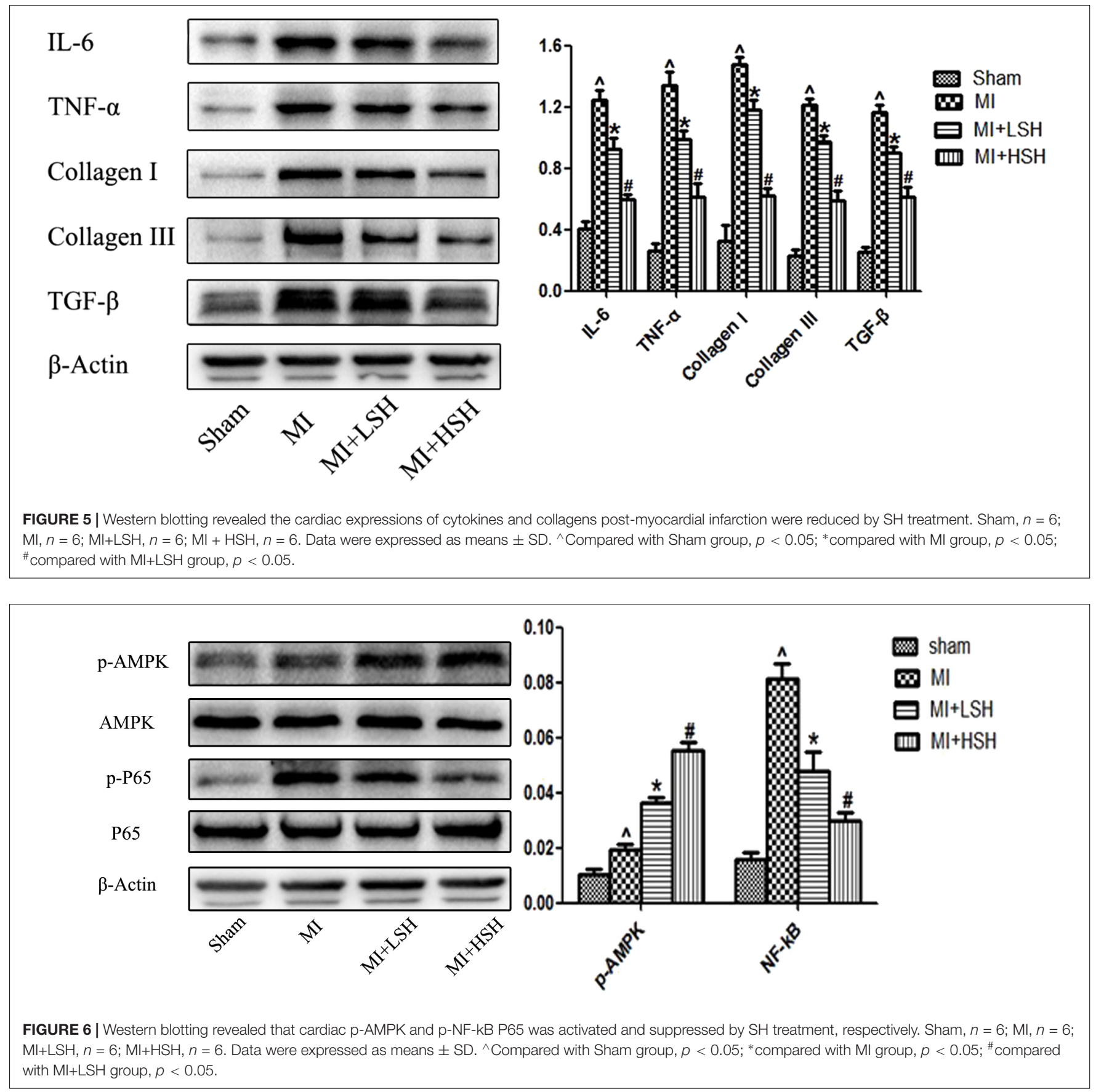

The infiltration of inflammation and fibrosis in left ventricle was visualized by HE stain and Mallory's trichrome stain, respectively. On the heart 28 day post-myocardial infarction, the infarcted zone became thin, mainly consisted of fibrotic scar tissue and inflammatory cells, few cardiomyocytes could be seen in this area. In the peri-infarcted zone, a large amount fibrotic tissue and inflammatory cells was found inserting into the cardiomyocytes. The remote zone dominantly consisted of cardiomyocytes, interspersed with a few fibrotic tissue and inflammatory cells. As showed in Figures 3, 4, treatment of SH alleviated the infiltration of inflammation and fibrosis in all these three zones. Moreover, the inflammatory and fibrotic changes in left ventricle were more suppressed by high dose SH.

\section{Sodium Houttuyfonate Suppressed the Cardiac Expression of Inflammatory Cytokines and Collagen Post-myocardial Infarction}

To further confirm the effect of SH on cardiac inflammation and fibrosis post-myocardial infarction, the cardiac expressions of TNF- $\alpha$, IL- 6 , TGF- $\beta$, collagen I and collagen III were investigated 

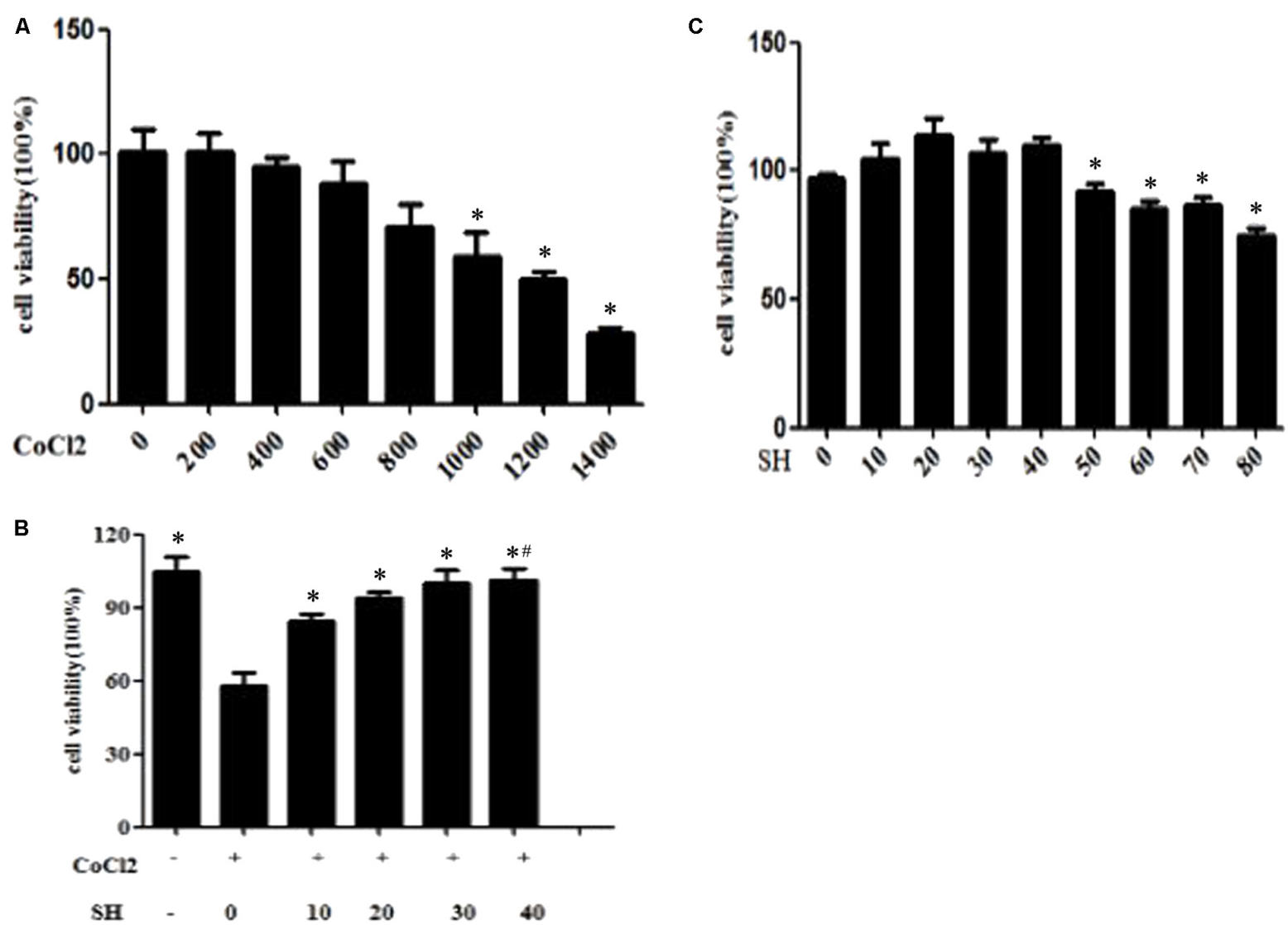

FIGURE 7 | CCK-8 assay showed that SH treatment improved the cell viability of $\mathrm{H} 9 \mathrm{C} 2$ post $\mathrm{CoCl}_{2}$-induced hypoxia. (A) $\mathrm{H} 9 \mathrm{C} 2$ were treated with various concentrations of $\mathrm{CoCl}_{2}(0,200,400,600,800,1000,1200$, and $1400 \mu \mathrm{M}, n=6$ in each concentration of CoCl $)$ alone for $24 \mathrm{~h}$, the concentration of $1000 \mu \mathrm{M}$ $\mathrm{CoCl}_{2}$ resulted in half cell death. *Compared with $0 \mu \mathrm{M} \mathrm{CoCl}$ treatment, $p<0.05$. (B) $\mathrm{H} 9 \mathrm{C} 2$ were treated with various concentrations of $\mathrm{SH}(0,10,20,30,40,50$, 60, 70, and $80 \mu \mathrm{g} / \mathrm{ml}, n=6$ in each concentration of SH) alone for $24 \mathrm{~h}$. *Compared with $0 \mu \mathrm{g} / \mathrm{mL} \mathrm{SH}$ treatment, $p<0.05$. (C) The H9C2 induced hypoxia by $\mathrm{CoCl}_{2}$ were treated with SH $\left(0,10,20,30\right.$, and $40 \mu \mathrm{g} / \mathrm{mL}, n=6$ in each concentration of SH) for $24 \mathrm{~h}$, respectively. ${ }^{*}$ Compared with $0 \mu \mathrm{g} / \mathrm{mL} \mathrm{SH}$ treatment, $p<0.05$. "Compared with $30 \mu \mathrm{g} / \mathrm{ml} \mathrm{SH}$ treatment, $p>0.05$.

by western blotting and compared among the four groups. As shown in Figure 5, compared with Sham group, there was obvious elevation of TNF- $\alpha$, IL- 6 , TGF- $\beta$, collagen I and collagen III in MI group $(p<0.05)$. SH treatment dose-dependently suppressed the expression of TNF- $\alpha$, IL- 6 , TGF- $\beta$, collagen I and collagen III and $(p<0.05)$, with high dose SH exhibiting a more notable inhibitory effect $(p<0.05)$.

\section{Sodium Houttuyfonate Treatment Activated AMPK and Suppressed NF-кB p65 Post-myocardial Infarction}

As the AMPK signal pathway was critically involved in the adaptive response to hypoxic and ischemic stress, and $H$. cordata has been reported could elicit and enhance the activation of AMPK in other animal models of diseases, we further investigated whether there is an influence of SH on AMPK during post-infarct remodeling. As shown in the Figure 6, compared with Sham group, there was an increased phosphorylation of AMPK in MI group $(p<0.05)$. SH treatment further increased the phosphorylation of AMPK $(p<0.05)$, and a more enhanced phosphorylation of AMPK was observed in the MI+HSH group $(p<0.05)$. The NF- $\kappa \mathrm{B}$ signaling pathway, which was a classical pathway of inflammation, was recently demonstrated to promote cardiac remodeling after MI. We also investigated the $\mathrm{SH}$ effect on NF- $\mathrm{B}$ signal pathway during post-infarct remodeling. As shown in the Figure 6, compared with Sham group, there was an obviously increased phosphorylation of NF-кB p65 in MI group. However, the phosphorylation of NF- $\mathrm{B}$ p 65 was obviously inhibited in SH treatment groups $(p<0.05)$, and a much higher inhibitory effect NF- $\kappa$ B 65 was found in $\mathrm{MI}+\mathrm{HSH}$ group $(p<0.05)$.

\section{Sodium Houttuyfonate Exerted a Protective Effect on H9C2 Post-hypoxia via Activating AMPK and Suppressing NF-кB Pathway}

To further elucidate the mechanism underlying $\mathrm{SH}$ treatment, $\mathrm{H} 9 \mathrm{C} 2$ were induced hypoxia by $\mathrm{CoCl}_{2}$ to simulate myocardial infarction in animal model. As shown in Figure 7A, based on CCK-8 assay, the $\mathrm{CoCl}_{2}$ reduced cell viability in a 

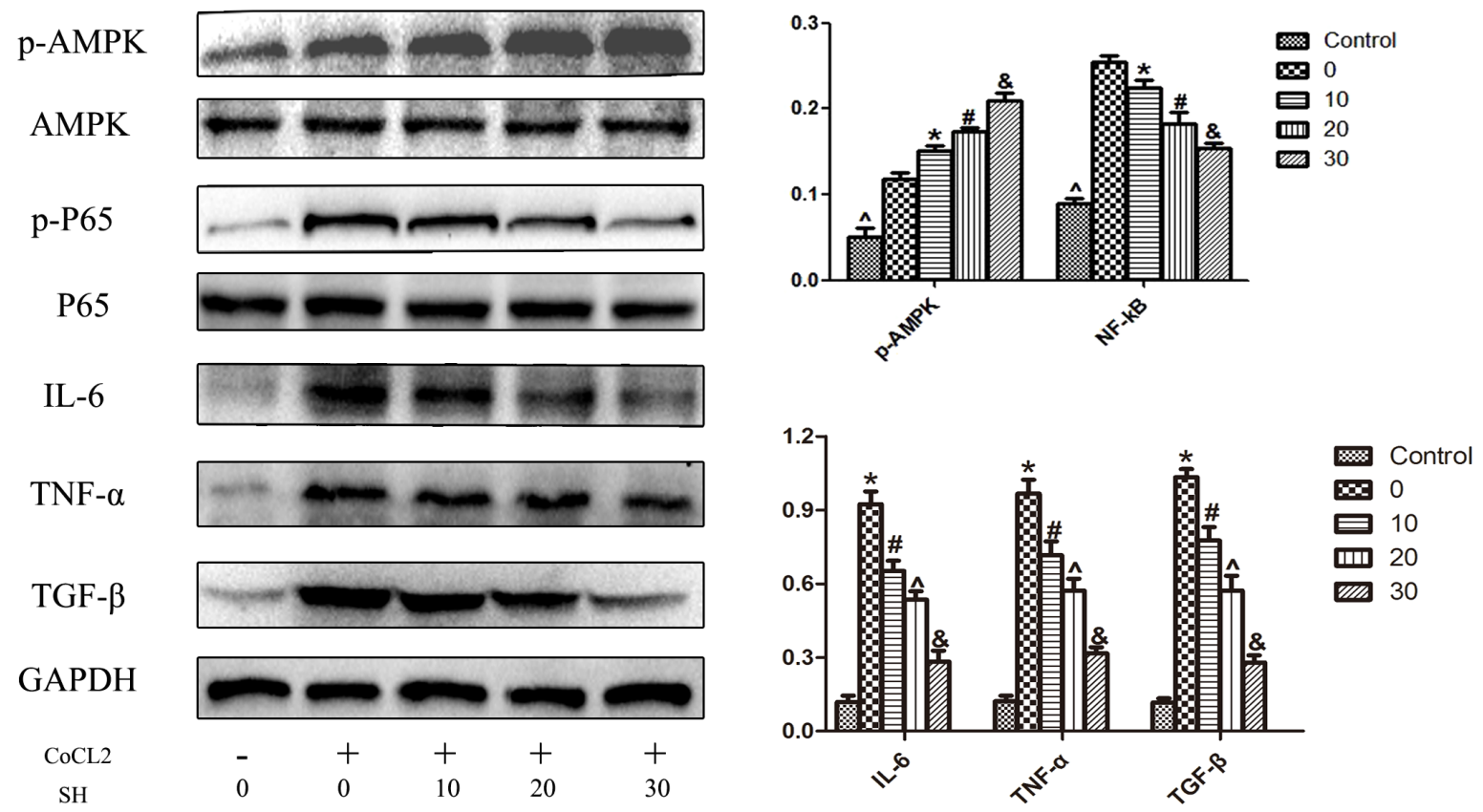

FIGURE 8 | Western blotting showed that SH decreased the expression of inflammatory cytokines, increased the activation of AMPK, suppressed the activation of NF-kB P65 on H9C2 post COCl 2 -induced hypoxia. Control, $n=6$; 0, $\mathrm{CoCl}_{2}+0 \mu / \mathrm{ml} \mathrm{SH}, n=6 ; 10, \mathrm{CoCl}_{2}+10 \mu \mathrm{g} / \mathrm{ml} \mathrm{SH}, n=6 ; 20, \mathrm{CoCl} 2+20 \mu \mathrm{g} / \mathrm{ml} \mathrm{SH}, n=6$; $30, \mathrm{CoCl}_{2}+30 \mu \mathrm{g} / \mathrm{ml}, n=6$. ${ }^{*}$ Compared with control, $p<0.05$; ${ }^{*}$ compared with $0 \mu \mathrm{g} / \mathrm{ml} \mathrm{SH}$ post $\mathrm{CoCl}_{2}, p<0.05 ;{ }^{\wedge}$ compared with $10 \mu \mathrm{g} / \mathrm{ml} \mathrm{SH}$ post CoCl 2 , $p<0.05 ;{ }^{*}$ compared with $20 \mu \mathrm{g} / \mathrm{ml}$ post $\mathrm{CoCl}_{2}, p<0.05$.

concentration-dependent manner and the concentration of $1000 \mu \mathrm{M}$ resulting in half cell death was applied to all cell experiments in this study. Meanwhile, H9C2 were treated with various concentrations of SH $(0-80 \mu \mathrm{g} / \mathrm{ml})$ alone for $24 \mathrm{~h}$. Cell viability did not change significantly when cells were treated with $\mathrm{SH}$ at different concentrations ranging from 10 to $40 \mu \mathrm{g} / \mathrm{ml}$ $(p>0.05)$, whereas SH at concentrations of $50-80 \mu \mathrm{g} / \mathrm{ml}$ induced slight cell viability reduction $(p<0.05)$, as shown in Figure 7B. The H9C2 cells induced chemical hypoxia by $1000 \mu \mathrm{M} \mathrm{CoCl} 2$ were then treated with $\mathrm{SH}(0,10,20,30$, and $40 \mu \mathrm{g} / \mathrm{ml})$ for $24 \mathrm{~h}$, respectively. In the range of $0-30 \mu \mathrm{g} / \mathrm{ml}$ $\mathrm{SH}, \mathrm{CoCl}_{2}$-induced cytotoxicity was inhibited and cell viability was enhanced in a dose-dependent manner $(p<0.05)$, as shown in Figure 7C. There was no significant changes in cell viability between administration of $30 \mu \mathrm{g} / \mathrm{ml}$ and $40 \mu \mathrm{g} / \mathrm{ml} \mathrm{SH}(p>0.05)$.

The effect of $\mathrm{SH}$ on $\mathrm{H} 9 \mathrm{C} 2$ post-hypoxia was further investigated by western blotting. As shown in Figure 8, in the range of $0-30 \mathrm{ng} / \mathrm{ml}$, SH decreased the expression of IL-6, TNF- $\alpha$ and TGF- $\beta$ in a dose-dependent manner $(p<0.05)$. The changes of AMPK and NF- $\mathrm{KB}$ signal pathway were also explored. In the range of $0-30 \mu \mathrm{g} / \mathrm{ml} \mathrm{SH}$ treatment, with the reduction of inflammatory cytokines, the AMPK and NF- $\mathrm{KB}$ p65 were gradually activated and suppressed. Whether there was a correlation between AMPK and NF- $\kappa B$ pathway under the treatment of $\mathrm{SH}$ post-hypoxia was further examined. After transfected with AMPKa siRNA, the expression of AMPK in H9C2 was remarkably down-regulated $(p<0.05)$. As shown in Figure 9, SH treatment post-hypoxia suppressed p-NF-кB p65 and inflammatory cytokines (IL-6, TNF- $\alpha$, and TGF- $\beta$ ) in H9C2 $(p<0.05)$. However, this protective effect of SH was reversed by AMPK knockdown. With the knockdown of AMPK, the NF- $\mathrm{B}$ p65 and inflammatory cytokines was significantly up-regulated $(p<0.05)$.

\section{DISCUSSION}

In the present study, the effect of $\mathrm{SH}$ on post-infarct remodeling was first and comprehensively investigated. We found that 4-week administration of SH post-myocardial infarction alleviated the adverse cardiac remodeling, ameliorated the inflammatory and fibrotic responses of cardiac tissue. Additionally, as treatment with SH increased AMPK activation and suppressed NF- $\mathrm{B}$ activation in our study, we suspected that the cardioprotective effect of $\mathrm{SH}$ post-myocardial infarction may be contributed to it regulating these signal pathways. To further explore the mechanism underlying the benefits of $\mathrm{SH}$ treatment, $\mathrm{H} 9 \mathrm{C} 2$ were induced hypoxia to simulate myocardial infarction in animal model, we found $\mathrm{SH}$ treatment reduced the expression of inflammatory cytokines, increased the activation of AMPK and decreased the activation of NF- $\mathrm{BB}$ on $\mathrm{H} 9 \mathrm{C} 2$ in a dose-dependent manner. Intriguingly, transfection H9C2 with AMPK $\alpha$ siRNA remarkably blunted the protective effect of SH post-hypoxia. Based on these findings, we concluded that $\mathrm{SH}$ treatment post-myocardial infarction could attenuate post-infarct remodeling, alleviate cardiac inflammatory and fibrotic responses, and the underlying mechanism was related to activating AMPK and suppressing NF- $\mathrm{BB}$ signal pathway. 
A

AMPK

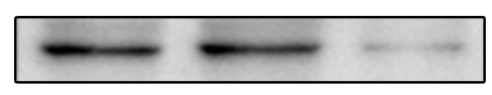

GAPDH

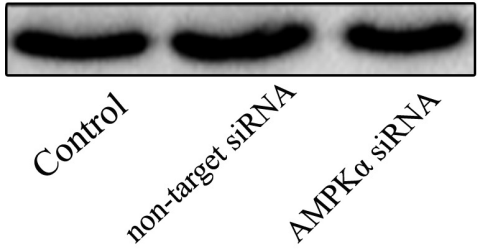

B

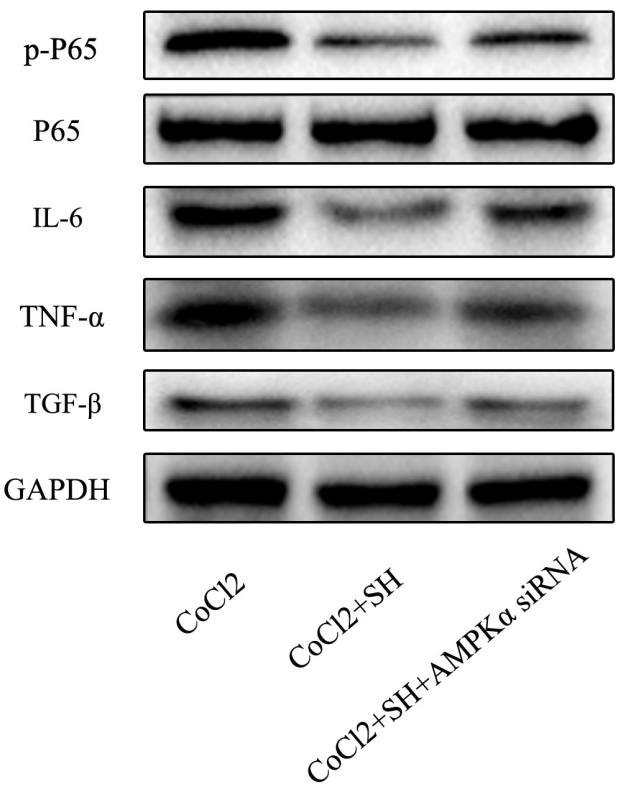

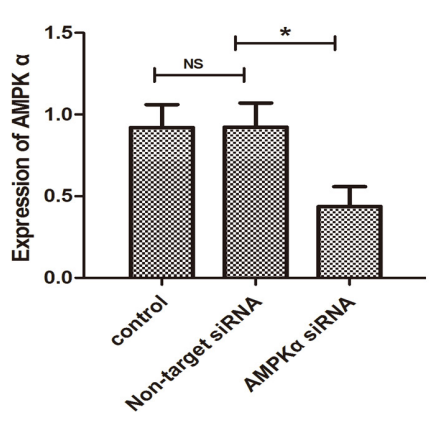
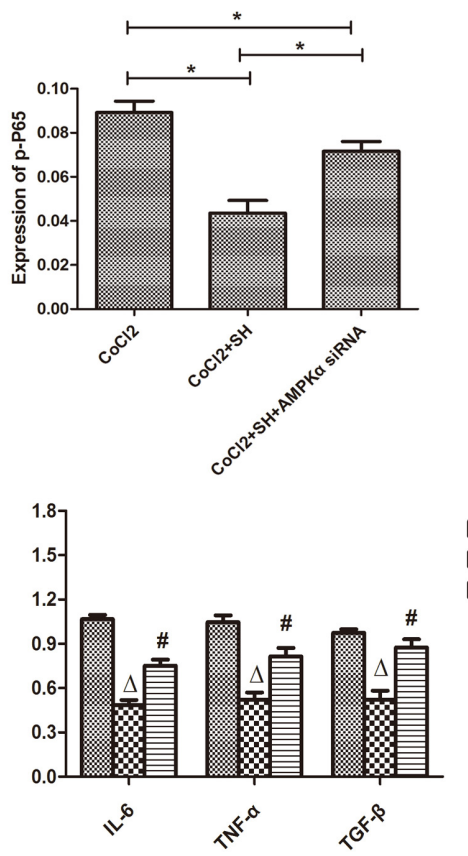

FIGURE 9 | Transfection H9C2 with AMPK $\alpha$ siRNA reversed the suppression of NF-kB p65 and pro-inflammatory cytokines by SH post-hypoxia. (A) The expression of AMPK of H9C2 transfected with AMPK $\alpha$ siRNA. Control, $n=6$; non-target siRNA, $n=6$; AMPK $\alpha$ siRNA, $n=6$. NS indicated $p>0.05$, *indicated $p<0.05$. (B) The expression of NF-kB p65, IL-6 and TNF $\alpha$ of H9C2 transfected with AMPK $\alpha$ siRNA. $\mathrm{CoCl}_{2}, n=6 ; \mathrm{CoCl}_{2}+\mathrm{SH}, n=6 ; \mathrm{CoCl}_{2}+\mathrm{SH}+\mathrm{AMPK}$ siRNA, $n=6$. *Indicated $p<0.05 ;{ }^{\Delta}$ compared with $\mathrm{CoCl}_{2}$ group, $p<0.05$; ${ }^{*}$ compared with $\mathrm{CoCl}_{2}+\mathrm{SH}$ group, $p<0.05$.

During past decades, with the widespread application of anti-remodeling drugs, angiotensin-converting-enzyme inhibitor (ACEI), angiotensin receptor blocker (ARB) and $\beta$-blocker, the prognosis of patients with myocardial infarction have been largely improved. Nevertheless, a part patient population post-myocardial infarction still developed into progressive cardiac remodeling and heart failure, due to no response to these anti-remodeling drugs or intolerance to the side effects caused by these agents (Gullo et al., 2014). Therefore, new and efficient anti-remodeling drugs are still urgently needed. Recent studies have revealed that post-infarct remodeling was associated with the persisting low-level inflammatory response and tissue fibrosis, moreover, modulation of the inflammatory and fibrotic responses could, to some extent, reverse the process of post-infarct remodeling (Li et al., 2006; Takahashi et al., 2008; Hamid et al., 2011; Sobirin et al., 2012; Shivshankar et al., 2014; Wang B. et al., 2017). H. cordata is a classical Chinese herb used in traditional Chinese medicine for inflammatory diseases, such as pneumonia. Recently, $\mathrm{SH}$, which was a pure compound extracted from $H$. cordata, has been found exerting anti-inflammatory and anti-fibrotic effects on animal and cell models of various diseases (Gao et al., 2010; Du et al., 2012; Kang and Koppula, 2014a, 2015; Xu et al., 2015; Zhu et al., 2015; Wu et al., 2017). On LPS-induced bovine endometrial epithelial cell inflammation, SH treatment significantly decreased the expression of pro-inflammatory cytokines and alleviated the inflammatory process. On chronic obstructive pulmonary disease (COPD) inflammatory model rats induced by combination usage of cigarette smoke and LPS, the pathological changes of lung tissues were considerably alleviated, as well as pro-inflammatory cytokines significantly decreased by $\mathrm{SH}$ treatment. $\mathrm{SH}$ treatment was also found decreasing TNF $\alpha$ level and attenuating ventricular remodeling induced by abdominal aortic banding in rats. On a rapid pulmonary fibrosis rat model, $\mathrm{SH}$ treatment repaired lung injury and reduced pulmonary fibrosis in a dose-dependent manner. As SH has been confirmed a potent anti-inflammatory 
and anti-fibrotic agent under diseased situations by previous studies, in this study, we aimed to investigate whether $\mathrm{SH}$ could exert an anti-remodeling effect post-myocardial infarction by alleviating inflammatory and fibrotic responses. Our study for the first time clarified that $\mathrm{SH}$ treatment can alleviate the post-infarct inflammation and fibrosis, reverse post-infarct remodeling and can be a promising drug therapy for chronic heart failure caused by myocardial infarction.

To elucidate the mechanism underlying $\mathrm{SH}$ treatment during post-infarct remodeling, the AMPK and NF- $\kappa$ B signal pathways were further investigated. It has been reported that AMPK was one of the downstream signal pathways of Cordata Houttuynia treatment under diseased situations (Kang and Koppula, 2014b; Wang J.H. et al., 2017). On the other hand, AMPK has been widely known involved in coordinating a series pathophysiological responses under ischemic stress, and pharmacological activation of AMPK has been found preventing inflammation and cellular death, reducing cardiac remodeling post-myocardial infarction (Calvert et al., 2008; Peairs et al., 2009; Chiang et al., 2017; Kim et al., 2017; Quan et al., 2018). In our study, we found that $\mathrm{SH}$ treatment elevated the activation of AMPK on post-infarct heart and post-hypoxia H9C2. Therefore, it was a reasonable guess that AMPK was critically involved in the anti-remodeling effect of $\mathrm{SH}$ post-myocardial infarction.

With the increased activation of AMPK pathway, enhanced suppression of NF- $\mathrm{B}$ pathway was also observed by $\mathrm{SH}$ treatment. Moreover, in $\mathrm{H} 9 \mathrm{C} 2$ post-hypoxia, knockdown of AMPK by siRNA blunted the suppression of $N F-\kappa B$ and inflammatory cytokines by $\mathrm{SH}$. Based on this observation, we proposed that the cardioprotective effect of $\mathrm{SH}$ was related to downregulating NF- $\mathrm{B}$ pathway by upregulating AMPK pathway. The NF- $\mathrm{B}$ signaling pathway, which was widely known as a classical pathway involved in inflammatory and fibrotic responses during various diseases, was recently demonstrated promoting adverse cardiac remodeling post-myocardial infarction by aggravating and prolonging the inflammatory and fibrotic processes (Martinez-Martinez et al., 2017; Wang B. et al., 2017). There were emerging evidences indicating AMPK signaling can inhibit the pathological responses induced by the nuclear factor- $\kappa \mathrm{B}(\mathrm{NF}-\kappa \mathrm{B})$ system. A plethora of studies revealed that AMPK did not suppress NF- $\kappa B$ signaling directly, its inhibition of NF- $\mathrm{B}$ was realized indirectly via its downstream mediators, e.g., Sirtuin-1 (SIRT1), Forkhead box $\mathrm{O}(\mathrm{F}$ oxO) family, and peroxisome proliferator-activated receptor $\gamma$ co-activator $1 \alpha$ (PGC-1 $\alpha)$ (Salminen et al., 2011). Based on these investigations, we proposed that $\mathrm{SH}$ treatment attenuated post-infarct remodeling and exerted cardioprotective effect via activating AMPK and suppressing NF- $\mathrm{B}$ pathway.

\section{REFERENCES}

Calvert, J. W., Gundewar, S., Jha, S., Greer, J. J., Bestermann, W. H., Tian, R., et al. (2008). Acute metformin therapy confers cardioprotection against myocardial infarction via AMPK-eNOS-mediated signaling. Diabetes 57, 696-705. doi: 10. 2337/db07-1098
In our study, by knockdown AMPK in H9C2, we confirmed the AMPK signal regulating NF- $\kappa$ B pathway during $\mathrm{SH}$ treatment under hypoxia. However, this relationship between AMPK and NF- $\kappa \mathrm{B}$ pathway under $\mathrm{SH}$ treatment was not further investigated in vivo. We once considered adopting $\mathrm{AMPK} \alpha$ knockout animal to testify the effect of $\mathrm{SH}$ on post-infarct remodeling. Whereas, it has been demonstrated that knockout of AMPK subunits in animal could result in cardiomyopathy and cardiac remodeling, which would complicate our investigation of $\mathrm{SH}$ on postinfarct remodeling (Dolinsky and Dyck, 2006; Sung et al., 2015). In addition, though the effect of SH treatment posthypoxia was only tested on cardiomyoblast H9C2 but not on cardiac fibroblast, it still can give a reasonable explanation of SH's anti-inflammatory and anti-fibrotic potent observed on myocardial infarcted rat. We have confirmed the $\mathrm{SH}$ reduced the proinflammatory cytokines (IL- 6 and TNF- $\alpha$ ) on H9C2 posthypoxia. Meanwhile, we also found that $\mathrm{SH}$ treatment lead to a significant reduction of TGF- $\beta$. TGF- $\beta$ has been already confirmed a central cytokine of fibrogenesis and play an essential role in the pathogenesis of fibrosis. A large amount studies have reported that after myocardial infarction, TGF- $\beta$ was markedly induced and rapidly activated in the infarcted myocardium (Dobaczewski et al., 2011). Upon TGF- $\beta$ stimulation, pathways related to tissue fibrosis were activated and collagen deposition was initiated during cardiac remodeling. With the reduction of TGF- $\beta$ by SH observed on H9C2 post-hypoxia, the anti-fibrotic effect of SH could be better understood.

\section{CONCLUSION}

In this study, we found $\mathrm{SH}$ treatment could alleviate postinfarct inflammatory and fibrotic responses, attenuate postinfarct remodeling in a dose-dependent manner. The underlying mechanism was associated with activation of AMPK and suppression of NF- $\kappa$ B pathway.

\section{AUTHOR CONTRIBUTIONS}

$\mathrm{CZ}$ and $\mathrm{X}-\mathrm{WL}$ designed and drafted the original research. $\mathrm{CZ}$, J-FL, Z-HL, ST, W-QL, Y-ZL, HL, Z-RL, and J-HC performed the experiments. All authors approved it for publication.

\section{FUNDING}

This research was funded by grant 81570299 from the National Natural Science Foundation of China.

Chiang, M. C., Nicol, C. J., and Cheng, Y. C. (2017). Resveratrol activation of AMPK-dependent pathways is neuroprotective in human neural stem cells against amyloid-beta-induced inflammation and oxidative stress. Neurochem. Int. 115, 1-10. doi: 10.1016/j.neuint.2017.10.002

Dobaczewski, M., Chen, W., and Frangogiannis, N. G. (2011). Transforming growth factor (TGF)-beta signaling in cardiac remodeling. 
J. Mol. Cell. Cardiol. 51, 600-606. doi: 10.1016/j.yjmcc.2010. 10.033

Dolinsky, V. W., and Dyck, J. R. (2006). Role of AMP-activated protein kinase in healthy and diseased hearts. Am. J. Physiol. Heart Circ. Physiol. 291, H2557-H2569. doi: 10.1152/ajpheart.00329.2006

Du, S., Li, H., Cui, Y., Yang, L., Wu, J., Huang, H., et al. (2012). Houttuynia cordata inhibits lipopolysaccharide-induced rapid pulmonary fibrosis by upregulating IFN-gamma and inhibiting the TGF-betal/Smad pathway. Int. Immunopharmacol. 13, 331-340. doi: 10.1016/j.intimp.2012.03.011

Gao, J. P., Chen, C. X., Wu, Q., Gu., W. L., and Li, X. (2010). Effect of sodium houttuyfonate on inhibiting ventricular remodeling induced by abdominal aortic banding in rats. Can. J. Physiol. Pharmacol. 88, 693-701. doi: 10.1139/ y10-049

Gullo, C. E, de Almeida Zia, V. A., and Vilela-Martin, J. F. (2014). Blockade of renin angiotensin system in heart failure post-myocardial infarction: what is the best therapy? Recent Pat. Cardiovasc. Drug Discov. 9, 28-37.

Hamid, T., Guo, S. Z., Kingery, J. R., Xiang, X., Dawn, B., and Prabhu, S. D. (2011). Cardiomyocyte NF-kappaB p65 promotes adverse remodelling, apoptosis, and endoplasmic reticulum stress in heart failure. Cardiovasc. Res. 89, 129-138. doi: $10.1093 / \mathrm{cvr} / \mathrm{cvq} 274$

Kang, H., and Koppula, S. (2014a). Hepatoprotective effect of houttuynia cordata thunb extract against carbon tetrachloride-induced hepatic damage in mice. Indian J. Pharm. Sci. 76, 267-273.

Kang, H., and Koppula, S. (2015). Houttuynia cordata alleviates high-fat dietinduced non-alcoholic fatty liver in experimental rats. Pharm. Biol. 53, 414-422. doi: $10.3109 / 13880209.2014 .923002$

Kang, H., and Koppula, S. (2014b). Houttuynia cordata attenuates lipid accumulation via activation of AMP-activated protein kinase signaling pathway in HepG2 cells. Am. J. Chin. Med. 42, 651-664. doi: 10.1142/ S0192415X14500426

Kim, N., Jung, Y., Nam, M., Sun Kang, M., Lee, M. K., Cho, Y., et al. (2017). Angiotensin II affects inflammation mechanisms via AMPK-related signalling pathways in HL-1 atrial myocytes. Sci. Rep. 7:10328. doi: 10.1038/s41598-01709675-3

Li, Y., Takemura, G., Okada, H., Miyata, S., Maruyama, R., Li, L., et al. (2006). Reduction of inflammatory cytokine expression and oxidative damage by erythropoietin in chronic heart failure. Cardiovasc. Res. 71, 684-694. doi: 10. 1016/j.cardiores.2006.06.003

Martinez-Martinez, E., Buonafine, M., Boukhalfa, I., Ibarrola, J., FernandezCelis, A., Kolkhof, P., et al. (2017). Aldosterone target NGAL (Neutrophil Gelatinase-Associated Lipocalin) is involved in cardiac remodeling after myocardial infarction through NFkappaB pathway. Hypertension 70, 1148-1156. doi: 10.1161/HYPERTENSIONAHA.117.09791

Peairs, A., Radjavi, A., Davis, S., Li, L., Ahmed, A., Giri, S., et al. (2009). Activation of AMPK inhibits inflammation in MRL/lpr mouse mesangial cells. Clin. Exp. Immunol. 156, 542-551. doi: 10.1111/j.1365-2249.2009.03924.x

Quan, N., Wang, L., Chen, X., Luckett, C., Cates, C., Rousselle, T., et al. (2018). Sestrin2 prevents age-related intolerance to post myocardial infarction via AMPK/PGC-1alpha pathway. J. Mol. Cell. Cardiol. 115, 170-178. doi: 10.1016/ j.yjmcc.2018.01.005
Salminen, A., Hyttinen, J. M., and Kaarniranta, K. (2011). AMP-activated protein kinase inhibits NF-kappaB signaling and inflammation: impact on healthspan and lifespan. J. Mol. Med. 89, 667-676. doi: 10.1007/s00109-011-0748-0

Shivshankar, P., Halade, G. V., Calhoun, C., Escobar, G. P., Mehr, A. J., Jimenez, F., et al. (2014). Caveolin-1 deletion exacerbates cardiac interstitial fibrosis by promoting M2 macrophage activation in mice after myocardial infarction. J. Mol. Cell. Cardiol. 76, 84-93. doi: 10.1016/j.yjmcc.2014.07.020

Sobirin, M. A., Kinugawa, S., Takahashi, M., Fukushima, A., Homma, T., Ono, T., et al. (2012). Activation of natural killer T cells ameliorates postinfarct cardiac remodeling and failure in mice. Circ. Res. 111, 1037-1047. doi: 10.1161/ CIRCRESAHA.112.270132

Sung, M. M., Zordoky, B. N., Bujak, A. L., Lally, J. S., Fung, D., Young, M. E., et al. (2015). AMPK deficiency in cardiac muscle results in dilated cardiomyopathy in the absence of changes in energy metabolism. Cardiovasc. Res. 107, 235-245. doi: $10.1093 / \mathrm{cvr} / \mathrm{cvv} 166$

Takahashi, K., Fukushima, S., Yamahara, K., Yashiro, K., Shintani, Y., Coppen, S. R., et al. (2008). Modulated inflammation by injection of high-mobility group box 1 recovers post-infarction chronically failing heart. Circulation 118(14 Suppl.), S106-S114. doi: 10.1161/CIRCULATIONAHA.107.757443

Wang, B., Li, L., Jin, P., Li, M., and Li, J. (2017). Hesperetin protects against inflammatory response and cardiac fibrosis in postmyocardial infarction mice by inhibiting nuclear factor kappaB signaling pathway. Exp. Ther. Med. 14, 2255-2260. doi: 10.3892/etm.2017.4729

Wang, J. H., Bose, S., Lim, S. K., Ansari, A., Chin, Y. W., Choi, H. S., et al. (2017). Houttuynia cordata facilitates metformin on ameliorating insulin resistance associated with gut microbiota alteration in oletf rats. Genes 8:E239. doi: 10. 3390/genes8100239

Wu, Z., Tan, B., Zhang, H., Guo, Y., Tu, Y., Qiu, F., et al. (2017). Effects of sodium houttuyfonate on pulmonary inflammation in COPD model rats. Inflammation 40, 2109-2117.doi: 10.1007/s10753-017-0650- 1

Xu, Y. Y., Zhang, Y. Y., Ou, Y. Y., Lu, X. X., Pan, L. Y., Li, H., et al. (2015). Houttuyniacordata Thunb. polysaccharides ameliorates lipopolysaccharideinduced acute lung injury in mice. J. Ethnopharmacol. 173, 81-90. doi: 10.1016/ j.jep.2015.07.015

Zhu, Q., Xu, X., Liu, X., Lin, J., Kan, Y., Zhong, Y., et al. (2015). Sodium houttuyfonate inhibits inflammation by blocking the MAPKs/NF-kappaB signaling pathways in bovine endometrial epithelial cells. Res. Vet. Sci. 100, 245-251. doi: 10.1016/j.rvsc.2015.04.004

Conflict of Interest Statement: The authors declare that the research was conducted in the absence of any commercial or financial relationships that could be construed as a potential conflict of interest.

Copyright () 2018 Zheng, Lin, Lin, Lin, Thapa, Lin, Lian, Liu, Chen and Li. This is an open-access article distributed under the terms of the Creative Commons Attribution License (CC BY). The use, distribution or reproduction in other forums is permitted, provided the original author(s) and the copyright owner(s) are credited and that the original publication in this journal is cited, in accordance with accepted academic practice. No use, distribution or reproduction is permitted which does not comply with these terms. 